\title{
Cochlear Implantation through the Middle Fossa Approach: A Review of Related Temporal Bone Studies and Reported Cases
}

\author{
Juan Carlos Cisneros Lesser ${ }^{1,2}$ Rubens Vuono de Brito Neto ${ }^{1}$ Graziela de Souza Queiroz Martins ${ }^{1}$ \\ Ricardo Ferreira Bento ${ }^{1}$ \\ ${ }^{1}$ Department of Otorhinolaryngology, Hospital das Clínicas, School of \\ Medicine, Universidade de São Paulo, HC-FMUSP, São Paulo, SP, Brazil \\ 2 Department of Otorhinolaryngology, Instituto Nacional de \\ Rehabilitación, Mexico City, Mexico \\ Address for correspondence Juan Carlos Cisneros Lesser, MD, Rua \\ Francisco Leitão 104, apt. 24. Pinheiros, São Paulo, SP, \\ Zip code: 05414-020, Brazil (e-mail: juancarloscisneros@me.com). \\ Int Arch Otorhinolaryngol 2017;21:102-108.
}

Abstract

Keywords
- cochlear implants
- deafness
- sensorineural hearing
loss
- middle fossa
approach

Introduction Middle fossa approach has been suggested as an alternative for patients in whom other routes of electrode insertion are contraindicated. Even though there are temporal bone studies about the feasibility of introducing the cochlear implant through the middle fossa, until now, very few studies have described results when cochlear implant surgery is done through this approach.

Objective The objective of this study is to review a series of temporal bone studies related to cochlear implantation through the middle fossa and the results obtained by different surgical groups after cochlear implantation through this approach.

Data Sources PubMed, MD consult and Ovid-SP databases.

Data Synthesis A total of 8 human cadaveric temporal bone studies and 6 studies reporting cochlear implant surgery through the middle fossa approach met the inclusion criteria. Temporal bone studies show that it is feasible to perform cochlear implantation through this route. So far, only two surgical groups have performed cochlear implantation through the middle fossa with a total of 15 implanted patients. One group entered the cochlea in the most upper part of the basal turn, inserting the implant in the direction of the middle and apical turns; meanwhile, the other group inserted the implant in the apical turn directed in a retrograde fashion to the middle and basal turns. Results obtained in both groups were similar.

Conclusions The middle fossa approach is a good alternative for cochlear implantation when other routes of electrode insertion are contraindicated.

\section{Introduction}

Cochlear implantation is an effective method for the rehabilitation of patients with severe and profound sensorineural hearing loss. The great technological advances in cochlear implants and the good auditory results obtained have widened the selection criteria for candidacy. Hence, there is great involvement from surgeons to develop different and less

received

January 12, 2016

accepted

February 16, 2016

published online

April 8, 2016 traumatic electrode insertion techniques. Simple mastoidectomy with a posterior tympanotomy is considered the classic route for cochlear implant surgery and it is still the most practiced technique. Other less common techniques for cochlear implantation include the suprameatal and transcanal approaches, both offering good results. In cases of patients with chronic suppurative otitis media, unstable mastoid cavities with recurrent otorrhea, partially ossified
Copyright $(2017$ by Thieme-Revinter

Publicações Ltda, Rio de Janeiro, Brazil
License terms

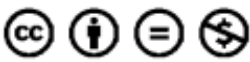


cochleae, and in some cases of inner ear dysplasia, the classic route, as well as the suprameatal and transcanal routes for cochlear implant insertion might be contraindicated. Middle fossa approach has been proposed as a good alternative in the cases mentioned earlier and an important number of temporal bone studies related to the anatomical basis of cochlear implantation through the middle fossa have been produced. Although a great number of papers describing the hearing results with cochlear implantation through the more common routes have been published, there are very few studies describing hearing results when cochlear implant surgery is done through a middle fossa approach and, apparently, only 15 patients around the world have been implanted through this approach. The objective of this study is to review a series of temporal bone studies related to cochlear implantation through the middle fossa and the results obtained by different surgical groups after attempting cochlear implantation through this approach.

\section{Review of the Literature}

\section{Search Strategy and Study Selection}

We conducted a systematic search in PubMed, MD consult, and Ovid SP databases from 1970 up to September 25, 2015, by combining the search terms "cochlear implant" and "cochlear implantation" with the terms "middle fossa," "middle fossa surgery," and "middle fossa approach" in the title and abstract fields. We checked the bibliography of all relevant articles to identify supplemental studies.

We identified 8 human cadaveric temporal bone studies with information relevant to cochlear identification for cochlear implantation through the middle fossa approach. One of the studies was excluded since it had the same information as another but published in another journal with a different language. Studies referring to middle fossa anatomy but without relevance to cochlear implantation surgery are not included in the review.

According to patients being implanted through a middle fossa approach, we found 6 studies in international literature. Four of the studies were done by the same group (Colleti et al) who wrote about their results in a total of 11 patients and another study was done by Bento et al who report the results in 4 more patients. The sixth study found is of Polish origin and only the abstract is available in the English language. It refers to one patient implanted through the middle fossa with good results, but since the full text of this article and relevant data are not available, it was not included in the review.

\section{Identifying the Cochlea through the Middle Fossa Approach}

The reasons for performing a middle fossa approach include, amongst others, surgery for schwannoma, meningioma and other petroclival tumors, resurfacing or obliteration of the superior semicircular canal for superior semicircular canal dehiscence syndrome, and the exploration and decompression of the labyrinth segment of the facial nerve. An important number of studies have focused on identifying landmarks to perform a safe middle fossa approach, since, due to differences in pneumatization between temporal bones, there are important variations in middle fossa anatomy. In 1998, Wysocki and Skarzynski ${ }^{1}$ studied 100 human temporal bones aged between 1 month and 71 years to identify anatomical landmarks related to cochlear implantation. They performed a microscopic dissection to expose the cochlear turns and its neighboring structures (internal carotid artery, jugular vein bulb, facial nerve, and internal acoustic meatus) measuring the minimal distances between them. They found that only children less than 4 years of age had significantly smaller and constant measured distances between the different structures and the cochlea compared with older children and adults. This suggests that the postnatal pneumatization of the petrous apex through aging has an important implication in the position of the different structures.

Probably the main reason for knowing how to identify the cochlea through the middle fossa is to properly avoid it, thus preventing hearing loss when accessing the internal ear canal for other purposes. In 2002, Bento et $\mathrm{al}^{2}$ described a safe technique for exposing the geniculate ganglion and the labyrinthine portion of the facial nerve without damaging the cochlea through the middle cranial fossa. This was done by exploring the roof of the middle ear and opening the tegmen tympani as a means to identify constant landmarks such as the cochleariform process. They described typical landmarks to identify not only the basal turn of the cochlea but the position of the apical turn. In 2004, Jung et $\mathrm{al}^{3}$ reported a morphometric analysis of the cochlea and its relationship with other middle fossa landmarks from 16 human cadaveric heads (32 temporal bones). The aim of this study was to give landmarks to minimize cochlear injury and preserve hearing during a middle fossa approach. Their measures showed an average cochlea-geniculate ganglion distance of $3.0 \mathrm{~mm}$, ranging from 1.2 to $4.1 \mathrm{~mm}$. The average cochlea-petrous carotid genu distance, cochlea-internal acoustic meatus distance, and cochlea-mandibular nerve distance were $2.9 \mathrm{~mm}, 9.0 \mathrm{~mm}$, and $9.4 \mathrm{~mm}$, respectively. In 2012, Forbes et $\mathrm{al}^{4}$ performed a study in which they recorded anatomic measurements describing the location of the cochlea in relation to the intersection of the greater superficial petrosal nerve (GSPN) and facial nerve from 11 cadaveric specimens dissected by means of an anterior petrosectomy and cochlear skeletonization. Then, they compared and correlated these measurements with thin-cut temporal bone computed tomography scans from 25 patients with morphologically normal inner ears. The authors concluded that the drilling of the anteromedial petrous bone outside of a radius of $12.5 \mathrm{~mm}$ from the extrapolated junction of GSPN and facial nerve is associated with a low degree of risk to damaging the cochlea.

Some authors have also proposed the identification of the basal turn of the cochlea through the middle fossa and its relations with adjacent structures as a means to insert a cochlear implant through this route. For instance, in 2004, Anagnostopoulou and Diamantopoulou ${ }^{5}$ did a study on 116 temporal bones with the objective of showing the anatomic relationships of the superior aspect of the cochlea with the adjacent superficial structures seen in the anterior surface of 
the petrous bone, to suggest a basis for an alternative approach to the cochlear turns through the middle cranial fossa considering cochlear implantation. They used the groove for the GSPN and the facial hiatus as landmarks to expose the superior aspect of the cochlea. They described that the position of the cochlea in the middle fossa floor is reliably an area medial to the first genu of the facial nerve and posterior to the lateral part of the GSPN. Interestingly, according to their measurements, the uppermost point of the basal turn of the cochlea may be projected onto the floor of the middle cranial fossa at the crossing point of two lines drawn on the anterior surface of the petrous bone, one being a line parallel and posterior to the course of the GSPN at an anteroposterior mean distance of $3.73 \mathrm{~mm}$ and the second being a line parallel and medial to the sagittal plane that corresponds to the medial wall of the first genu of the facial canal at a mean distance of $2.60 \mathrm{~mm}$.

In 2007, Todd $^{6}$ reported a study in 82 temporal bones in which he inserted commercially available Med-El (Medical Electronics, Innsbruck, Austria) split arrays through the middle fossa. By X-ray, he assessed mastoid size, depth of insertion, and electrode placement. In every bone, electrode insertion was accomplished both anterior-medially, toward the round window, and posterior-laterally, toward the cochlear apex. In his study, more than $75 \%$ of the electrodes inserted toward the round window extended into the vestibule, whereas insertions toward the cochlear apex had a median insertion depth of $12 \mathrm{~mm}$ (range $6 \mathrm{~mm}$ to $18 \mathrm{~mm}$ ), allowing electrode access to nearly the full length of the cochlea.

Rubens de Brito et al, ${ }^{7,8}$ in 2013, published a study of 50 temporal bones with the objective of describing a novel approach for exposing the basal turn of the cochlea through the middle fossa for cochlear implantation. They used the superior petrosal sinus, the skeletonized petrous apex, the lateral surface of the meatal plane trailed in its most proximal portion, and the GSPN as landmarks to perform a cochleostomy in the basal turn of the cochlea and cochlear implantation in direction of the apex (oriented toward the arcuate eminence direction). They measured the mean distance between the cochleostomy and the round window $(8.38 \pm 1.96 \mathrm{~mm})$ as well as the distance between the cochleostomy and the other landmarks ( $9.19 \pm 1.59 \mathrm{~mm}$ to the superior petrosal sinus and a mean minor distance of $6.63 \pm 1.38 \mathrm{~mm}$ and major distance of $8.29 \pm 1.43 \mathrm{~mm}$, to the most proximal portion of the long axis of the meatal plane). They tested electrode insertions with a fake electrode and observed that the electrode array accessed almost the full length of the cochlea and suggested that insertion through the scala tympani could be done with ease through the technique proposed.

\section{Cochlear Implantation Results When Done through the Middle Fossa Approach}

In 1998 , Colleti et $\mathrm{al}^{9}$ published the first of a series of 4 papers referring to cochlear implantation through the middle fossa. They described the surgical technique and the very early results of the first two patients with bilateral sensorineural hearing loss and middle ear disease implanted through a middle fossa cochleostomy. The first patient had a bilateral radical mastoidectomy and the second an adhesive otitis media. After exposing the middle cranial fossa floor, they identified the superior aspect of the basal turn of the cochlea by drilling an area positioned in the angle between the greater superficial petrous nerve and the facial nerve. They performed a cochleostomy no bigger than $1.5 \mathrm{~mm}$ on the most superficial part of the basal turn of the cochlea and inserted the cochlear implants in the direction of the apical turn of the cochlea. The receiver stimulator was positioned in a niche previously drilled in the squamous portion of the temporal bone. The first patient received a Nucleus CI24M (Cochlear Corporation, Sydney, Australia) and the second a LAURA-Flex implant (Philips Heering Implants, Antwerp, Belgium). The position of the cochlear implants toward the cochlear apex was documented by computed tomographic scan in both patients. They saw remarkable improvement in the outcome of speech perception scores after 30 days of the activation versus the preoperative values.

In 1999, the same group reported the results obtained in a total of 9 adult patients implanted through the middle fossa approach (which included the two reported earlier but with a longer follow-up) in two different publications. ${ }^{10,11}$ All patients had profound bilateral hearing loss and their etiologies included a bilateral radical mastoidectomy cavity and an adhesive otitis media (from the first published paper), autoimmune inner ear disease, previous cranial trauma, genetic pre-lingual deafness, and otosclerosis. All seven new cases underwent surgery in the same fashion as the first two reported. A Nucleus 24M (Cochlear Corporation) was inserted in 3 more patients; a Lauraflex implant (Philips Hearing Implants) was used in two more patients and a Combi $40+$ double electrode array (Med-El) in the last two. All single electrode arrays were inserted in the direction of the cochlear apex and double electrode arrays were inserted, with one electrode toward the apex and the other toward the round window. Telemetry and intraoperative recording of electrically-evoked auditory brainstem responses (EABR) was satisfactory in all patients. Speech recognition tests, during a period of time ranging from one to six months after activation, were obtained and found to be even better than those obtained in postlingually deaf patients operated with a traditional transmastoid route. The authors posted special attention to the possibility of stimulating the whole cochlea with the double array electrodes. In the year 2000 , the fourth paper from Colleti et $\mathrm{al}^{12}$ showed the results of the 9 patients mentioned earlier but with a longer follow-up and 2 other patients who had bilateral genetic pre-lingual deafness for a total of 11 patients. The results obtained, types of implants used, cause of deafness, and followup are summarized in - Table 1. In their series, they noticed no relevant postsurgical complications.

In 2012, Bento et $\mathrm{al}^{13}$ performed a retrospective case review study in which they report the results of cochlear implantation via the middle fossa approach in 4 patients with bilateral profound hearing loss, discussing the complications they found as well as a description of the programming considerations for the 4 cases. They indicated middle fossa approach, since 3 patients had bilateral radical "unstable" mastoid cavities and one had tympanic membrane perforations with bad tympanoplasty results. As a surgical technique, after appropriate exposure 
Table 1 Patient description and results obtained with cochlear implantation through the middle fossa by two surgical groups

\begin{tabular}{|c|c|c|c|c|c|c|c|c|c|}
\hline \multicolumn{10}{|c|}{ Patient description and results obtained } \\
\hline$\#$ & Author & Gen & $\begin{array}{l}\text { Age } \\
\text { (y) }\end{array}$ & Cause of deafness & $\begin{array}{l}\text { Dur } \\
\text { (y) }\end{array}$ & Implant / side & Follow-up & $\begin{array}{l}\text { Free-field } \\
\text { hearing } \\
\text { threshold } \\
\text { average }\end{array}$ & $\begin{array}{l}\text { Open set } \\
\text { sentence } \\
\text { recognition } \\
(\%)\end{array}$ \\
\hline 1 & \multirow[t]{11}{*}{$\begin{array}{l}\text { Colleti } \\
\text { et al }\end{array}$} & M & 58 & $\begin{array}{l}\text { Fibro-adhesive } \\
\text { otitis media }\end{array}$ & $3 \mathrm{R} 6 \mathrm{~L}$ & Laura-Flex / L & 9 months & $N / A^{* *}$ & $90 \%$ \\
\hline 2 & & M & 39 & Head trauma & $18 \mathrm{R} 10 \mathrm{~L}$ & Laura-Flex / L & 6 months & $N / A^{* *}$ & $75 \%$ \\
\hline 3 & & M & 20 & Genetic (prelingual) & $20 \mathrm{R} 20 \mathrm{~L}$ & Laura-Flex / R & 6 months & $N / A^{* *}$ & $50 \%$ \\
\hline 4 & & $\mathrm{M}$ & 66 & Chronic ear disease & $12 \mathrm{R} 40 \mathrm{~L}$ & Nucleus CI24M / R & 9 months & $\mathrm{N} / \mathrm{A}^{* *}$ & $85 \%$ \\
\hline 5 & & $\mathrm{~F}$ & 19 & Cogan syndrome & $2 \mathrm{R} 2 \mathrm{~L}$ & Nucleus CI24M / R & 6 months & $N / A^{* *}$ & $75 \%$ \\
\hline 6 & & $\mathrm{M}$ & 18 & Genetic (prelingual) & $18 \mathrm{R} 18 \mathrm{~L}$ & Nucleus CI24M / L & 6 months & $\mathrm{N} / \mathrm{A}^{* *}$ & $70 \%$ \\
\hline 7 & & $M$ & 23 & Genetic (prelingual) & $23 \mathrm{R} 23 \mathrm{~L}$ & Nucleus CI24M / R & 1 month & $N / A^{* *}$ & $40 \%$ \\
\hline 8 & & M & 57 & Otosclerosis & $4 \mathrm{R} 4 \mathrm{~L}$ & Nucleus CI24M / L & 3 months & $N / A^{* *}$ & $60 \%$ \\
\hline 9 & & M & 24 & Cogan syndrome & $0.5 \mathrm{R} 0.5 \mathrm{~L}$ & $\begin{array}{l}\text { Combi } 40+\text { double } \\
\text { array / } R\end{array}$ & 6 months & $\mathrm{N} / \mathrm{A}^{* *}$ & $75 \%$ \\
\hline 10 & & $\mathrm{~F}$ & 9 & Genetic (prelingual) & $9 \mathrm{R} 9 \mathrm{~L}$ & $\begin{array}{l}\text { Combi } 40+\text { double } \\
\text { array / L }\end{array}$ & 3 months & $N / A^{* *}$ & $30 \%$ \\
\hline 11 & & M & 13 & Genetic (prelingual) & $13 \mathrm{R} 13 \mathrm{~L}$ & $\begin{array}{l}\text { Combi } 40+\text { double } \\
\text { array / L }\end{array}$ & 1 month & $N / A^{* *}$ & $35 \%$ \\
\hline 12 & \multirow[t]{4}{*}{$\begin{array}{l}\text { Bento } \\
\text { et al }\end{array}$} & M & 38 & Chronic ear disease & $4 \mathrm{R} 4 \mathrm{~L}$ & $\begin{array}{l}\text { Nucleus } 24 \text { R } \\
\text { (ST) / L }\end{array}$ & $\begin{array}{l}{ }^{*} \text { mean } \\
23 \text { months }\end{array}$ & $36.25 \mathrm{~dB}$ & $100 \%$ \\
\hline 13 & & $\mathrm{~F}$ & 61 & Chronic ear disease & $6 \mathrm{R} 5 \mathrm{~L}$ & $\begin{array}{l}\text { Nucleus } 24 \text { RE } \\
\text { (ST) / L }\end{array}$ & $\begin{array}{l}\text { *mean } \\
23 \text { months }\end{array}$ & $37.5 \mathrm{~dB}$ & $30 \%$ \\
\hline 14 & & $\mathrm{~F}$ & 46 & $\begin{array}{l}\text { Ototoxicity and } \\
\text { chronic ear disease } \\
\text { secondary to } \\
\text { radiotherapy }\end{array}$ & $6 \mathrm{R} 6 \mathrm{~L}$ & $\begin{array}{l}\text { Nucleus } 24 \text { RE } \\
\text { (ST) / R }\end{array}$ & $\begin{array}{l}{ }^{*} \text { mean } \\
23 \text { months }\end{array}$ & $38.3 \mathrm{~dB}$ & $90 \%$ \\
\hline 15 & & $\mathrm{~F}$ & 46 & Chronic ear disease & $26 \mathrm{R} 26 \mathrm{~L}$ & $\begin{array}{l}\text { Med-El Sonata } \\
\text { Medium / L }\end{array}$ & $\begin{array}{l}{ }^{*} \text { mean } \\
23 \text { months }\end{array}$ & $47.5 \mathrm{~dB}$ & $50 \%$ \\
\hline
\end{tabular}

Abbreviations: F, female; M, male; Gen, gender; y, years; Dur, duration of deafness before implantation; L, left ear; R, right ear.

Note: The cause of deafness is described as well as the time of deafness before implantation. The implanted side and implant model used are described. ${ }^{*}$ In the article by Bento et al, ${ }^{13}$ there is no specific description of the follow-up time, only the mean for the 4 patients.

${ }^{* *}$ Colleti et $\mathrm{l}^{9-12}$ do not describe free field hearing thresholds.

of the middle fossa floor, they drilled out a triangular bony area between the GSPN and the projection of the labyrinthine portion of the facial nerve and the geniculate ganglion. They took care not to damage the fundus of the internal auditory meatus. Different from what Colleti et al proposed, ${ }^{9-12}$ they entered the cochlea with a $1 \mathrm{~mm}$ drill at the most superior part of the apical turn and inserted the electrode array in a reversed direction, starting at the apex and passing through the middle turn to the basal turn of the cochlea. The authors suggested that the closer the cochleostomy is to geniculate ganglion, the higher the chance of a correct apical insertion. Intraoperative impedance recordings were normal in 3 patients with one having two open electrodes and neural response telemetry was performed with satisfactory responses in two of the patients, with one presenting facial nerve stimulation. ACT scan verified the correct electrode insertion in all four patients. Three patients received a Nucleus 24 cochlear implant system (Cochlear Corporation) and one received a Sonata Medium device (Med-El). A niche in the squamous temporal bone was drilled to place the receiver stimulator in the same fashion as used for the conventional transmastoid approach. In this series, full insertion was achieved in only one patient who received one of the Nucleus 24 cochlear implants. Considering the electrode design and cochlear tonotopicity, the frequency allocation tables were reversed. Every patient tested the maps with reversed and conventional programming, and after a time of experience, they reported which was better. In the end, two patients ended using the reversed map and two patients the conventional. Hearing thresholds showed that the four patients gained good access to speech perception with a mean follow-up of 23 months. The data from this study is also summarized in - Table 1 together with the results from Colleti et $\mathrm{al}^{9-12}$ for comparison.

\section{Discussion}

Temporal bone studies have shown that it is possible to perform a safe cochlear implant insertion through the middle fossa in spite of its difficult anatomy. When reviewing the 
results obtained by the surgical groups mentioned earlier, middle fossa approach for cochlear implant insertion appears to be a valid alternative, especially for patients with chronic suppurative otitis media and unstable mastoid cavities with recurrent otorrhea. The more common way to perform cochlear implantation in the presence of cholesteatoma or chronic otorrhea is through staged operations. The primary surgery is done to eradicate infection and cholesteatoma. If an open cavity is created, some type of obliteration has to be performed, either with an abdominal fat graft or a temporalis muscle flap. The secondary surgery is for implant insertion and it is generally performed 6 to 12 months after the first surgical procedure. ${ }^{14,15}$ Cochlear implantation through the middle fossa approach is a single step surgery, thus, providing benefit in this group of patients.

In the series by Colleti et $\mathrm{al}^{9-12}$ two out of the eleven patients implanted through the middle fossa had an actual indication to avoid the transmastoid classic route. The authors state that the speech perception outcomes in the first two patients with chronic ear disease and the ease and absence of complications of the technique induced them to use the middle fossa approach in 9 more patients without middle ear disease. They suggest that patients with inner ear malformations can also benefit greatly from the middle fossa approach by avoiding contamination from the middle ear, which poses a greater risk of meningitis, as well as to avoid electrode penetration into the internal auditory canal.

Bento et $\mathrm{al}^{13}$ state that cochlear implantation through the middle cranial fossa is challenging even for experienced surgeons, as it requires a highly precise technique with limited exposure, landmark identification is difficult, and there is an important risk of facial nerve damage. The risk of facial nerve damage seems to be greater with the surgical technique proposed by Bento et al. ${ }^{13}$ They state that the closer the cochleostomy is to geniculate ganglion, the higher the chance for a correct apical insertion. It is the authoŕs opinion that this particular risk must be reduced when approaching the cochlea through the basal turn instead of the apical turn. The anatomical temporal bone study by Brito et $\mathrm{al}^{7}$ shows a practical and safe way to approach the basal turn of the cochlea through the middle fossa approach as well as an intuitive way to introduce the electrode array in the correct fashion, with an orientation toward the middle and apical turns.

In terms of programming, it seems that it is easier for audiologists to perform the cochlear implant when the device is placed in a typical route, starting in the upper part of the basal turn of the cochlea and directing the electrode to the apex, rather than attempting insertion through the apical turn. The normal route avoids the problem of considering reversed frequency allocation to stimulate according to normal cochlear tonotopicity. Still, the results obtained in the cases when the cochlear implant was inserted in the "reversed" fashion were comparable to those obtained with a basal to apical oriented insertion or with a double array.

The use of double array implants showed results similar to those obtained with a single electrode. One must remember that in the temporal bone study performed by Todd, ${ }^{6} 75 \%$ of the electrodes directed from the cochleostomy to the round window extended into the vestibule, which may be dangerous in a real clinical scenario by promoting electrically induced disequilibrium in patients with some preserved vestibular function.

Finally, it is important to mention that middle fossa surgery carries its own risks, as described by Bento et al $^{16}$ and that this surgical approach should only be performed by experienced surgical teams.

\section{Final Comments}

Cochlear implantation through the middle cranial fossa is an alternative that provides a good possibility for patients with open cavities and profound hearing loss. It may provide results that are comparable to results obtained with a traditional insertion route in selected patients. It is the authoŕs opinion that this procedure should only be performed by experienced surgeons and that it should not be considered the first choice for patients that can be managed with a more traditional insertion route. In most cases the classic transmastoid approach with posterior tympanotomy should still be considered the first choice for cochlear implant insertion.

Note

The authors declare no conflicts of interest.

\section{References}

1 Wysocki J, Skarzyñski H. Distances between the cochlea and adjacent structures related to cochlear implant surgery. Surg Radiol Anat 1998;20(4):267-271

2 Bento RF, de Brito RV, Sanchez TG. A rapid and safe middle fossa approach to the geniculate ganglion and labyrinthine segment of the facial nerve. Ear Nose Throat J 2002;81(5):320-326

3 Jung SM, Jang JS, Ahn TH. Microanatomical study of the extradural middle fossa approach for preventing cochlear damage. J Korean Neurosurg Soc 2004;36:353-357

4 Forbes JA, Rivas A, Tsai B, et al. Microsurgical localization of the cochlea in the extended middle fossa approach. J Neurol Surg B Skull Base 2012;73(6):410-414

5 Anagnostopoulou S, Diamantopoulou P. Topographic relationship between the cochlea and the middle fossa floor: the anatomical basis for an alternative approach to the cochlear turns. Surg Radiol Anat 2004;26(2):82-85

6 Todd NW. Cochlear implantation via the middle fossa: surgical and electrode array considerations. Cochlear Implants Int 2007;8(1): $12-28$

7 Brito Rd, Bittencourt AG, Tsuji RK, Magnan J, Bento RF. Cochlear implantation through the middle fossa: an anatomic study for a novel technique. Acta Otolaryngol 2013;133(9):905-909

8 Bittencourt AG, Tsuji RK, Tempestini JPR, Jacomo AL, Bento RF, Brito Rd. Cochlear implantation through the middle cranial fossa: a novel approach to access the basal turn of the cochlea. Braz J Otorhinolaryngol 2013;79(2):158-162

9 Colletti V, Fiorino FG, Carner M, Pacini L. Basal turn cochleostomy via the middle fossa route for cochlear implant insertion. Am J Otol 1998;19(6):778-784

10 Colletti V, Fiorino FG. New window for cochlear implant insertion. Acta Otolaryngol 1999;119(2):214-218 
11 Colletti V, Fiorino FG, Saccetto L, Giarbini N, Carner M. Improved auditory performance of cochlear implant patients using the middle fossa approach. Audiology 1999;38(4):225-234

12 Colletti V, Fiorino FG, Carner M, Sacchetto L, Giarbini N. New approach for cochlear implantation: cochleostomy through the middle fossa. Otolaryngol Head Neck 66 Surg 2000;123(4):467-74

13 Bento RF, Bittencourt AG, Goffi-Gomez MV, Samuel P, Tsuji RK, de Brito R. Cochlear implantation via the middle fossa approach: surgical and programming considerations. Otol Neurotol 2012; 33(9):1516-1524
14 Leung R, Briggs RJ. Indications for and outcomes of mastoid obliteration in cochlear implantation. Otol Neurotol 2007; 28(3):330-334

15 El-Kashlan HK, Arts HA, Telian SA. External auditory canal closure in cochlear implant surgery. Otol Neurotol 2003; 24(3):404-408

16 Bento RF, Pirana S, Brito RV, Sanchez TG, Castilho AM, Sweet RC. O papel do acesso via fossa média no tratamento da paralisia facial traumática. Rev Bras Otorinolaringol 2004;70(4): 484-493 
